\title{
Chronic vascular effects of oat phenolic acids and avenanthramides in pre- or stage 1 hypertensive adults
}

\author{
Gulten Soycan ${ }^{1}$, Manuel Y. Schar ${ }^{1}$, Giulia Corona $^{2}$, Angelika Kristek ${ }^{1}$, Sarah Alsharif ${ }^{1}$, \\ Jodee Johnson ${ }^{3}$, Peter Shewry ${ }^{4}$ and Jeremy Paul Edward Spencer ${ }^{1}$ \\ ${ }^{1}$ University of Reading, Reading, United Kingdom, \\ ${ }^{2}$ University of Roehampton, London, United Kingdom, \\ ${ }^{3}$ Quaker Oats Center of Excellence, PepsiCo R\&D Nutrition Sciences, Illinois, USA and \\ ${ }^{4}$ Rothamsted Research, Harpenden, United Kingdom
}

\begin{abstract}
Wholegrain consumption is linked to a lower risk of cardiovascular disease. Evidence from randomized controlled trials have established that the consumption of wholegrain oats lowers blood cholesterol, via a mechanism partly mediated by $\beta$-glucan soluble fiber. However, oats contain an array of phenolic acids, including ferulic acid and also structurally related avenanthramides, which may also contribute to the cardiovascular health benefits of oat intake. We investigated whether 4 weeks, daily consumption of oat phenolics leads to improvement in markers of CVD risk men and women.In a 3 arm crossover single-blind, placebo-controlled trial, 28 volunteers consumed either: 1) oatmeal/oatcake intervention (-containing $48.9 \mathrm{mg}$ of phenolic acids and $19.2 \mathrm{mg}$ of avenanthramides); 2) oatbran concentrate + rice porridge/wheat cracker intervention (-containing $38.4 \mathrm{mg}$ of phenolic acids and $0.5 \mathrm{mg}$ of avenanthramides) or 3) rice porridge/wheat cracker intervention (containing $13.8 \mathrm{mg}$ of phenolic acids). All treatments were matched in soluble fiber $(4.8 \mathrm{~g})$ and energy $(500 \mathrm{kcal})$. The primary endpoint was FMD and other cardiovascular endpoints were blood pressure, LDI, LDL/HDL cholesterol, platelets and endothelial cell-derived extracellular vesicles (EVs). All measures were taken at baseline and after three, 4 week long intervention periods and two washout periods.Our data indicates an increase by $1.09 \% \pm 0.41 \%($ Mean \pm $\mathrm{SEM})$ in FMD response following high phenolic oat intake with a significant difference $(\mathrm{P}=0.007)$ between baseline and postintervention. Consumption of high phenolic oats also led to a significant improvement in 24-hour SBP, day time SBP and night time SBP $(\mathrm{P}<0.01, \mathrm{P}<0.01$ and $\mathrm{P}<0.05)$ and day time and night time DBP $(\mathrm{p}<0.05)$. There was also a significant decrease with total and LDL cholesterol after the consumption of moderate and high phenolic oat interventions $(\mathrm{P}<0.05)$ and a small improvement in LDI (both Ach and SNP) but not significant. The number of resting endothelial EVs were also found to be increasing after the consumption of high phenolic oats. The findings of this study may provide evidence about the role of oat phenolic acids and avenanthramides in cardiovascular health and contribute to more effective public health advice about the consumption of oats and healthy cardiovascular aging.
\end{abstract}

\section{Conflict of Interest}

Jodee Johnson is employee of PepsiCo, Inc., which manufactures oatmeal products under the brand name Quaker Oats. The views expressed in this poster are those of the authors and do not necessarily reflect the position or policy of PepsiCo, Inc 